COLABORACIÓN ESPECIAL

\title{
ACTIVIDADES PARA CAPTAR Y VACUNAR A LA POBLACIÓN SUSCEPTIBLE EN LA COMUNIDAD VALENCIANA
}

\author{
Rosa Martín-Ivorra, Ana Ma Alguacil-Ramos, José A Lluch-Rodrigo, Eliseo Pastor-Villalba \\ y Antonio Portero-Alonso.
}

Servicio de Salud Infantil y de la Mujer. Dirección General de Salud Pública. Conselleria de Sanitat. Valencia.

No se han recibido becas u otro tipo de subvenciones para la elaboración de este trabajo.

Los autores declaran haber participado en reuniones científicas y proyectos financiados por la industria farmacéutica relacionadas con las vacunaciones y haber recibido bolsas de viaje para asistencia a reuniones científicas.

\section{RESUMEN}

La Organización Mundial de la Salud en su revisión del Plan de eliminación del sarampión y la rubéola remarcó la necesidad de evaluar las coberturas vacunales y desarrollar estrategias para mejorarlas y eliminar las bolsas de baja cobertura vacunal. Existe evidencia del papel que pueden jugar los sistemas de información vacunal para mejorar estas coberturas.

El objetivo del presente artículo es describir las acciones realizadas a partir del Sistema de Información Vacunal de la Comunidad Valenciana en la mejora de las coberturas vacunales.

Se trata de un sistema integral que permite le gestión de los programas de vacunación. Está conectado con otros sistemas de manera que se facilita la toma de decisiones relacionadas con las vacunaciones, tanto clínicas como epidemiológicas o de gestión.

Respecto al papel que juega en la mejora de las coberturas vacunales, e Sistema de Información Vacunal facilita la realización de todas las alternativas que se han demostrado efectivas: cálculo de coberturas y difusión de resultados, actividades de captación activa, colaboración con los servicios de vigilancia epidemiológica, gestión de la logística de las vacunas.

Se puede concluir, por tanto, que el Sistema de Información Vacunal es una herramienta útil para mejorar las coberturas vacunales y contribuir así a la eliminación del sarampión y la rubéola.

Palabras clave: Sistema de información. Sarampión. Rubéola. Vacunación masiva. Vacunas. Vacuna antisarampión. Vacuna antirubeola. Vacuna contra el sarampión, parotiditis, rubeola.

Correspondencia

José A. Lluch-Rodrigo

Avda. Cataluña, 21.

46010 Valencia

Correo electrónico: 1luch_jos@gva.es

\section{ABSTRACT \\ Activities to catch up and Vaccinate the Susceptible Population in Valencian Community, Spain}

Eliminating measles and rubella and preventing congenital rubella infection plan of WHO highlighted the need to assess vaccination coverage and develop strategies to improve it and eliminate pockets of low immunization coverage. It exists evidence about the potential role of immunization information systems to improve coverage.

The aim of this article is to describe the actions taken from the Vaccine Information System of Valencian Community addressed to improve the vaccination coverage.

The Vaccine Information System is a comprehensive information system that allows the management of vaccination programs. It is connected to other systems so that decisions related to vaccinations as clinical and epidemiological management is facilitated.

Regarding the role in improving vaccination coverage, the Vaccine Information System facilitates the implementation of all alternatives that have been proven effective: calculate coverage and feedback of results, active recruitment, cooperate with the epidemiological surveillance and logistics management of vaccines.

We can conclude, therefore, that the Vaccine Information System is a useful tool to improve vaccination coverage and thus contribute to the elimination of measles and rubella.

Keywords: information system. Measles. Rubella. Vaccines. Vaccination. Mass vaccination. Measles-Mumps-Rubella Vaccine. Rubella vaccine. Measles vaccine.

DOI: 


\section{INTRODUCCIÓN}

El Plan para la eliminación del sarampión y la rubéola, y la prevención de la infección congénita por el virus de la rubéola ${ }^{1}$ establece entre otras medidas mantener una elevada cobertura vacunal frente al sarampión y la rubéola mediante la vacunación sistemática y la realización de actividades específicas de captación de grupos susceptibles.

En el año 2013 la OMS revisó la situación y, como parte de las estrategias, estableció reforzar la gestión de los programas de vacunación y la monitorización de las coberturas vacunales alcanzadas $^{2}$.

Recientemente la Oficina para la Región Europea de la OMS aprobó el Plan de Acción Europeo de Vacunación 2015-20203 en el que insiste en la necesidad de mantener altas coberturas vacunales y disponer de registros de calidad que permitan gestionar los programas de vacunación.

La utilidad de los sistemas de información vacunal en la mejora de las coberturas de vacunación ha sido ampliamente demostrada. En una revisión realizada por The Community Preventive Services Task Force incluyeron 240 artículos o resúmenes referidos a este tema ${ }^{4}$. En este análisis se encontró evidencia sobre la efectividad de los sistemas de información en:

- Apoyar intervenciones efectivas para aumentar las tasas de vacunación (sistemas de recordatorio de dosis a usuarios, retroalimentación de la información a los profesionales, etcétera.)

- Determinar el estado de vacunación de la población para facilitar la toma de decisiones clínicas, epidemiológicas o de gestión.

- Orientar las respuestas de salud pública a los brotes de enfermedades prevenibles por vacunación.

- Evaluación de la cobertura de vacunación, las oportunidades perdidas de vacunación, los errores programáticos y la calidad de la información.
- Facilitar la gestión y evaluación de los programas de vacunación.

El objetivo del presente artículo es describir las acciones realizadas en la mejora de las coberturas vacunales a partir del Sistema de Información Vacunal de la Comunidad Valenciana.

\section{CONTEXTO}

La Comunidad Valenciana (CV) contaba a fecha 1 de enero de 2014 con una población de 5.004.844 habitantes 5 , lo que la sitúa en una posición intermedia en cuanto a tamaño de la población entre Irlanda (4.605.501) y Noruega $(5.107 .970)^{6}$. Ese mismo año 2014 se produjo un total de 44.308 nacimientos ${ }^{5}$.

Todos las personas empadronadas en la $\mathrm{CV}$ cuentan con una tarjeta sanitaria que incluye un número de identificación personal (número SIP) que sirve como identificador en el sistema sanitario tanto para los documentos individuales como para los sistemas de registro informáticos ${ }^{7}$.

Desde el punto de vista de la organización sanitaria, la CV está dividida en 24 departamentos de salud (equivalentes a las áreas de salud) ${ }^{8}$ con un total de 245 zonas básicas, desde donde desarrollan su actividad los equipos de atención primaria. Además cuenta con un total de 17 centros de Salud Pública, cada uno de ellos coordina las actividades de salud pública en uno o varios departamentos de salud y entre sus funciones se encuentra la gestión de la vigilancia epidemiológica y de los programas de vacunación ${ }^{9}$.

Características del Sistema de Información Vacunal (SIV) de la Comunidad Valenciana

Según la Organización Mundial de la Salud se entiende por sistemas de información sanitaria la estructura para la recolección de datos, el proceso, el análisis y la transmisión de la información necesarios para organizar $\mathrm{y}$ hacer funcionar los servicios sanitarios ${ }^{10}$. 
La confidencialidad, seguridad, simplicidad y compatibilidad con otros sistemas de información sanitaria, flexibilidad, accesibilidad, exhaustividad y calidad de los datos que se recogen, destacan entre las características básicas que deben tener los registros de vacunaciones nominales ${ }^{11}$.

El SIV de la CV es un sistema de información nominal e integral que pretende dar respuestas a las necesidades de información relacionadas con los programas de vacunación tanto a nivel clínico individual como a nivel epidemiológico y de gestión ${ }^{12}$.

Tiene carácter nominal, es decir, es un registro informático basado en una base de datos poblacional en la que se declaran de manera individualizada las vacunas administradas a cada persona en la CV. Por otro lado tiene carácter general en el sentido que permite el registro de cualquier vacunación, independientemente de que esté o no incluida en los programas de vacunación de la Conselleria de Sanitat, de la edad de la persona vacunada y del tipo de centro desde el que se realiza la atención.

$\mathrm{El}$ acceso al SIV se hace siempre con un procedimiento seguro, bien mediante la utilización de usuario/contraseña o bien mediante firma electrónica. El acceso se puede realizar desde otras aplicaciones informáticas (por ejemplo desde la historia de salud de atención primaria) o directamente a través de una página web segura (https://rvn. sp.san.gva.es/portalSIV/).

Los usuarios del SIV tienen asignados metaperfiles y ámbitos geográficos específicos de manera que se delimita el acceso tanto a los diferentes módulos del SIV como al ámbito territorial de acuerdo con las funciones que tienen y sus lugares de trabajo.

Por otro lado, el SIV tiene un carácter multicomponente, integrando los siguientes módulos (figura 1):
- Registro de vacunas nominal, desde el que se pueden registrar las vacunas administradas, notificar los eventos adversos potencialmente ligados a una vacuna, consultar el historial vacunal individualizado y emitir el certificado correspondiente y, por último, obtener listados de población vacunada utilizando diferentes criterios (intervalos de fechas de vacunación o nacimiento, tipos de vacunas, dosis...).

- Registro de vacunas de almacén, que permite la gestión integral de la logística de las vacunas, gestión de las incidencias en la cadena de frío y gestión centralizada de los procedimientos de compra.

- Backoffice, que incluye tanto las herramientas de mantenimiento del sistema como las de consulta. También incluye las herramientas de captación activa de personas susceptibles de vacunación y consulta masiva de situación de vacunal en contactos de casos durante brotes epidémicos de enfermedades inmunoprevenibles.

- Indicadores, desde los que se pueden consultar indicadores básicos de los programas de vacunación desagregados por centro de vacunación, zona básica, departamento de salud y comunidad autónoma.

- Convi, diseñado para la gestión de las consultas de los centros de vacunación internacional dependientes de la Conselleria de Sanitat.

Por último, se trata de un sistema interconectado con otros sistemas de información y gestión (figura 2):

- Sistemas de información de recursos [Catálogo de Recursos Corporativos (CRC)] y poblacionales [Sistema de Información Poblacional (SIP)] desde donde se recoge la información registrada de los centros sanitarios y los datos poblaciones respectivamente. 


\section{Figura 1 \\ Módulos del Sistema de Información Vacunal de la Comunidad Valenciana}

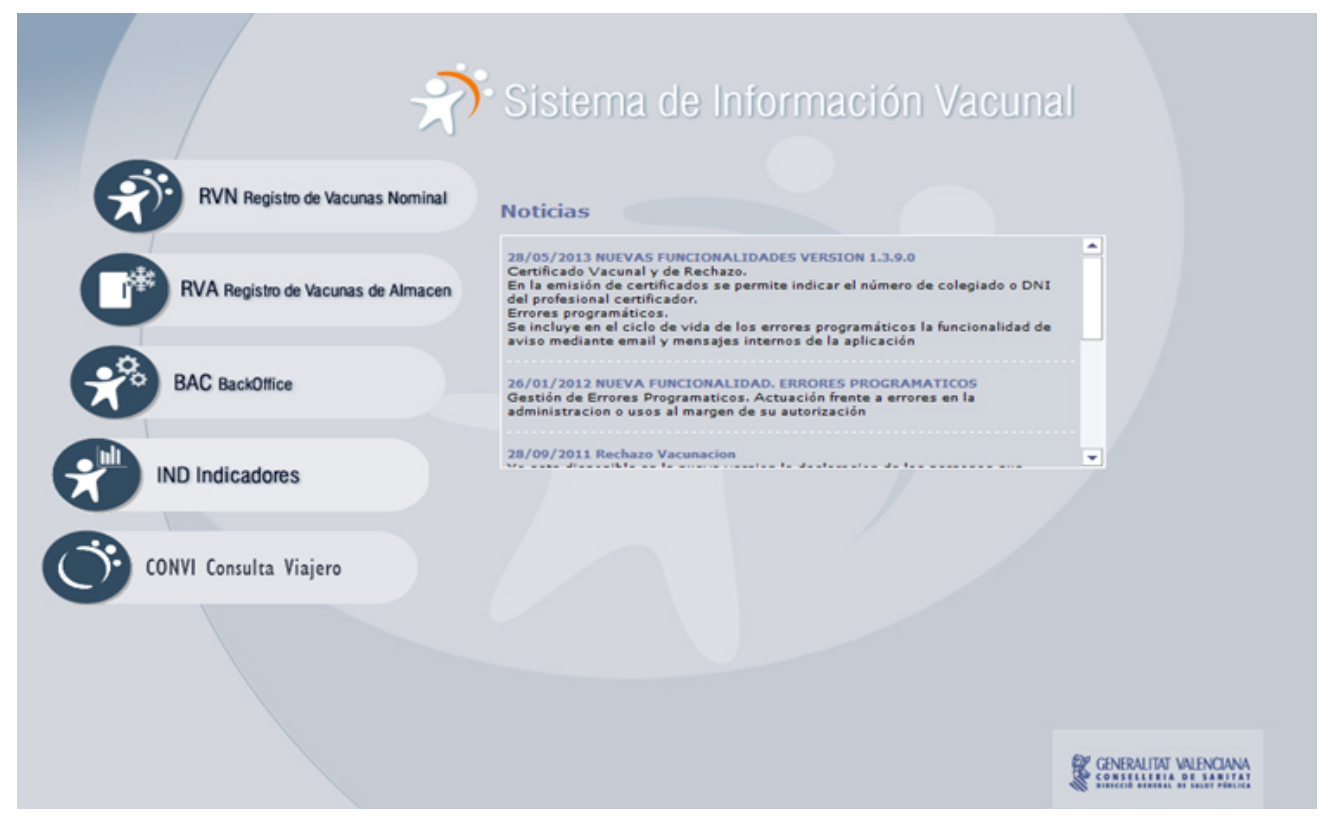

- Sistemas de información clínica [historia de salud de atención primaria, Sistema de Información Ambulatoria (SIA), registros hospitalarios: Orion-Clinic] a los que informa del historial vacunal individualizado y desde los que se pueden registrar las actividades de vacunación.

- Sistemas de gestión económica (COBRA, COMPAS).

- Sistema de farmacovigilancia, al que informa de los eventos adversos registrados como potencialmente relacionados con la vacunación.

- Sistema de control de exposición accidental a riesgos biológicos: Registro de Exposiciones Biológicas Accidentales (REBA), al que facilita información del estado vacunal de los accidentados.
- Sistemas de vigilancia epidemiológica [Análisis de Vigilancia Epidemiológica (AVE), Red de Vigilancia Microbiológica (RedMIVA)], a los que facilita información del estado vacunal de los casos de enfermedades inmunoprevenibles o de la población general para las actuaciones ante brotes de estas enfermedades.

A fecha 30 de junio de 2015 tenían acceso al SIV un total de 57.852 usuarios (1.178 usuarios no pertenecientes al sistema público) que accedían desde un total de 1.969 centros (670 centros privados). El número de actos vacunales registrados hasta esa fecha era de 32.076.257.

\section{ESTRATEGIAS PARA MEJORAR LAS COBERTURAS VACUNALES}

Las estrategias que se realizan para mejorar las coberturas vacunales en general y la 


\section{Figura 2}

\section{Esquema de la interconexión del SIV con otros sistemas de información}

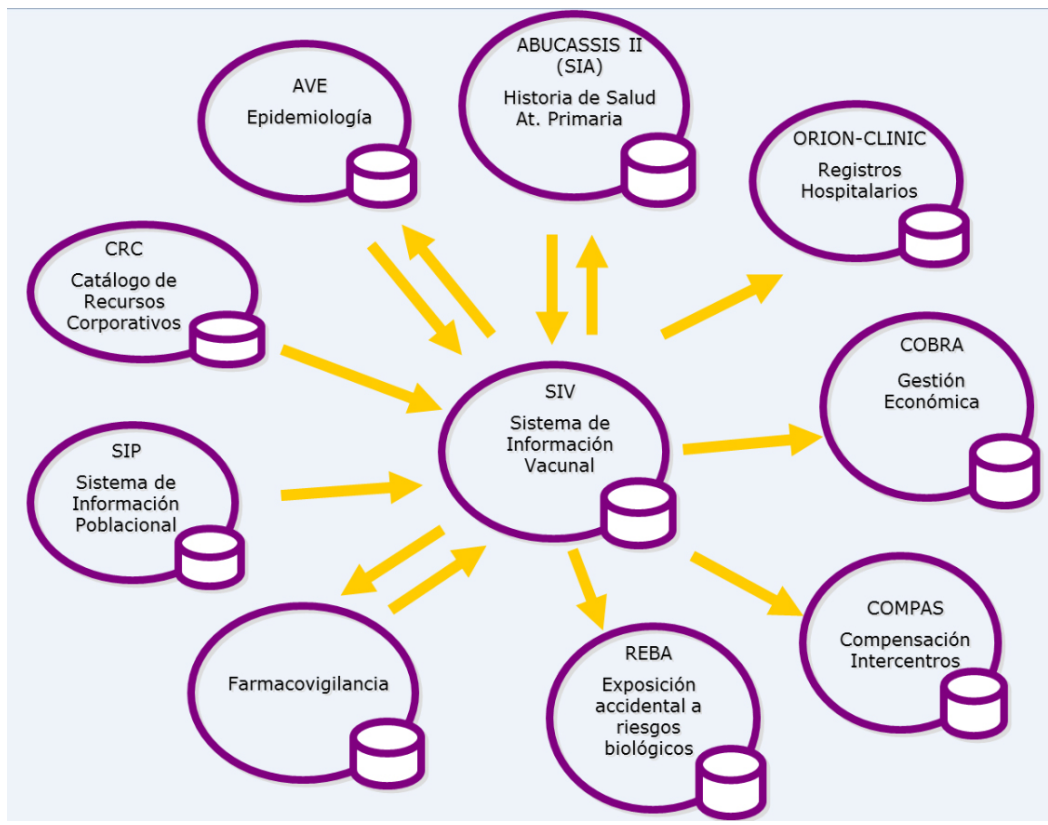

vacunación frente al sarampión y la rubéola en particular, en términos generales, son las que recoge The Community Preventive Services Task Force en su revisión"

\section{Retroalimentación de la información}

En primer lugar se realiza de forma sistemática el cálculo de coberturas para cada una de las etapas del calendario de vacunación infantil. Desde el módulo de indicadores del SIV se pueden consultar los indicadores básicos, mostrándose los datos de cobertura del centro desde el que se consulta, los correspondientes al departamento de salud donde se encuentra el centro y los de la CV.

Semanalmente se emite un informe que incluye las coberturas vacunales alcanzadas en cada uno de los departamentos de salud para la tercera dosis de vacunación frente a la poliomielitis, la primera dosis de vacunación con la vacuna triple vírica (sarampión, rubéola y parotiditis) y la cuarta dosis de vacuna frente a difteria, tétanos, tos ferina (DTP). Se muestran como diagramas de barras y una tabla con los datos y tiene como objetivo que los responsables de los departamentos de salud puedan hacer un seguimiento rápido de la situación respecto a la vacunación infantil.

En otros programas de vacunación, como la antigripal, se emiten informes similares con datos de cobertura de la temporada en curso y un análisis comparativo con la temporada anterior.

En la misma línea de devolución de resultados, trimestralmente se envía un fichero en formato de hoja de cálculo con la información detallada hasta zona básica de salud a los centros de salud pública para su difusión 
entre los responsables de vacunación. En las zonas básicas en las que las coberturas se encuentran por debajo de los objetivos marcados se realizan las intervenciones necesarias para mejorarlas.

\section{Captación activa}

Respecto a la captación activa el SIV dispone de un procedimiento de identificación de personas susceptible de ser vacunadas y de las que no se tiene constancia de que lo hayan sido. El sistema genera listados de personas con sus datos de identificación, localización (teléfono, dirección postal,...) y vacunas a las que se refiere el procedimiento. Este sistema permite emitir cartas de aviso automáticamente o realizar contacto telefónico con los usuarios para fomentar su vacunación. En el caso de la vacunación con triple vírica este procedimiento ha demostrado que es capaz de recuperar cerca del $35 \%$ de las personas avisadas $^{13}$.

También se elaboran listados de personas susceptibles de vacunación, por ejemplo de pacientes con diagnósticos clínicos donde esté indicada la vacunación antigripal, para facilitar la búsqueda activa.

Actualmente está en fase de desarrollo un procedimiento de alertas individualizado que permitiría al profesional sanitario identificar a las personas que acuden a las consultas programadas en las que estaría recomendada alguna vacuna en función de criterios personales, tales como edad, patología de base, estado vacunal previo, situaciones especiales individuales o comunitarias.

Intervención ante casos y brotes epidémicos

Desde el punto de vista de las actuaciones coordinadas con la vigilancia epidemiológica de las enfermedades inmunoprevenibles, los sistemas de la CV verifican de forma automatizada el estado vacunal registrado en el SIV de los pacientes diagnosticados de cualquiera de estas enfermedades.
Igualmente, con el fin de identificar a las personas previamente vacunadas cuando se está diseñando la intervención ante un brote epidémico, el SIV dispone de un procedimiento de consulta masiva del estado vacunal. De manera inmediata se puede conocer la situación vacunal respecto a una enfermedad inmunoprevenible de un listado de contactos.

Gestión de los programas de vacunación

El SIV recoge todos los aspectos necesarios para una correcta gestión de los programas de vacunación al integrar en los diferentes módulos toda la información necesaria.

A través del módulo de registro de almacén permite garantizar la calidad y la trazabilidad de las vacunas (control de existencias, registro de las incidencias en la cadena de frío,...) y el conocimiento de los costes de los programas (gestión de la facturación).

Facilita y simplifica la notificación de los posibles eventos adversos que se sospechen relacionados con la vacunación.

Permite también registrar las vacunas rechazadas por los usuarios.

\section{PRINCIPALES RETOS}

Se ha trabajado para afrontar los retos que se encuentran cuando se trabaja con sistemas de información de estas características.

En primer lugar, con el fin de incorporar la actividad de vacunación que se realiza en el ámbito de la asistencia sanitaria privada, se ha fomentado la formalización de acuerdos de colaboración con los centros privados. Entre los 670 centros actualmente adheridos a estos acuerdos se encuentran hospitales privados, consultas de pediatría o servicios de prevención de riesgos laborales. Los centros se comprometen a seguir los programas de vacunación de la Conselleria de Sanitat así como registrar la actividad vacunal en el SIV a cambio de recibir de forma gratuita las vacunas incluidas en estos programas. 
Respecto a la calidad de los datos, se realizan auditorias sistemáticas que tienen como objetivo garantizarla identificando, por ejemplo, las declaraciones duplicadas o los errores en el registro. En relación a la exhaustividad, otro de los puntos clave a considerar, se ha demostrado que en el SIV se registran más del $95 \%$ de las vacunas distribuidas y no incluidas de forma sistemática en los calendarios de vacunación ${ }^{14,15}$.

La dificultad de disponer de denominadores adecuados para el cálculo de coberturas de vacunación se minimiza en el caso del SIV, pues al utilizar datos nominales procedentes del sistema de información poblacional es posible no sólo calcular las coberturas en pequeños territorios sino incluso por clave médica.

Estos registros nominales permiten a su vez el cálculo de coberturas por cohortes de edad, lo que proporciona información sobre las vacunaciones realizadas fuera de los plazos establecidos y recomendados en los calendarios de vacunación.

También se tienen en cuenta los problemas relacionados con el rechazo a los programas de vacunación. Es posible registrar a través del SIV el rechazo a todas o a alguna de las vacunas, pudiendo identificar no sólo a los sujetos no vacunados a título individual sino también a posibles agrupaciones de no vacunados $\mathrm{y}$, de esta manera poder realizar estrategias de vigilancia y control de enfermedades específicas así como acciones de intervención informativas y educacionales.

Por último, si bien la vacunación de los niños es sumamente importante en el control de las enfermedades inmunoprevenibles, es necesario poder localizar bolsas de sujetos susceptibles de edades más avanzadas y definir estrategias para controlarlas. El SIV ayuda a identificar estas personas y a establecer estrategias de captación para mejorar las coberturas.

\section{BIBLIOGRAFÍA}

1. Oficina Regional para Europa de la OMS. Eliminación de sarampión y rubéola, y prevención de la infección congénita por el virus de la rubéola Plan estratégico 2005-2010 para la Región Europea de la OMS. (citado el 25/06/15). Disponible en: http://www.isciii.es/ISCIII/ es/contenidos/fd-servicios-cientifico-tecnicos/fd-vigilancias-alertas/fd-enfermedades/fd-enfermedadesprevenibles-vacunacion/OMSMeaslesStrategy2005.pdf

2. Oficina Regional para Europa de la OMS. Measles and rubella elimination 2015. Package for accelerated action: 2013-2015. (citado el 25/06/15). Disponible en: http://www.euro.who.int/_data/assets/ pdf_file/0020/215480/PACKAGE-FOR-ACCELERATED-ACTION-20132015.pdf?ua $=1$

3. Oficina Regional para Europa. European Vaccine Action Plan 2015-2020. (citado el 25/06/15). Disponible en: http://www.euro.who.int/_data/assets/pdf_ file/0007/255679/WHO_EVAP_UK_v30_WEB-newsticker.pdf?ua=1

4. Groom H, Hopkins DP, Pabst LJ, Murphy Morgan $\mathrm{J}$, Patel M, Calonge $\mathrm{N}$ et al. Immunization Information Systems to Increase Vaccination Rates: A Community Guide Systematic Rev J Public Health Manag Pract. 2015 May-Jun;21(3):227-48.

5. Instituto Nacional de Estadística. Padrón Municipal 1 de enero de 2014. (citado el 26/06/15). (citado el 26/06/15). Disponible en: http://www.ine.es/jaxiT3/ Datos.htm?t=2853

6. Eurostat Population on 1 January. (citado el 1/07/15). Disponible en: http://ec.europa.eu/eurostat/tgm/table. do $?$ tab $=$ table \&init $=1 \&$ language $=$ en $\&$ pcode $=$ tps 00001 \&plugin $=1$

7. Diari Oficial de la Comunitat Valenciana. Llei 6/2008 de Aseguramiento Sanitario. Valencia: DOCV núm 5778 de 05/06/2008. Disponible en: http://www. san.gva.es/documents/151744/225990/LeydeAseguramientoSanitario2008_6867.pdf

8. Diari Oficial de la Comunitat Valenciana. Llei 10/2014, de 29 de desembre, de la Generalitat, de Salut de la Comunitat Valenciana. DOCV núm. 7434 de 31/12/2014. Disponible en: http://www.docv.gva.es/ datos/2014/12/31/pdf/2014_11888.pdf

9. Diari Oficial de la Comunitat Valenciana. Orden 10/2012, de 24 de julio, de la Conselleria de Sanidad, por la que se procede a la actualización de la Cartera de Servicios de Salud Pública de la Comunitat Valenciana. DOCV núm. 6866 de 20/09/2012. Disponible en: http://www.docv.gva.es/portal/ficha_disposicion_ pc.jsp?sig $=008657 / 2012 \& \mathrm{~L}=1$

10. Wordl Health Oorganization. Health Information System. Report on a Conference. Copenhagen: WHO; 1973. 
11. Pastor E. Las nuevas tecnologías de la información en los registros vacunales. IV Simposio Intercongresos de la Asociación Española de Vacunología; 14 y 15 de Noviembre de 2008. Logroño: Asociación Española de Vacunología; 2008. (consultado el 12/07/15). Disponible en: http://www.vacunas.org/es/info-profesionales/temasdel-mes/tm-anteriores/9918-las-nuevas-tecnologias-dela-informacion-en-los-registros-vacunales?lang=es

12. Pastor-Villalba E, Martín-Ivorra R, Alguacil-Ramos AM, Portero-Alonso A, Lluch-Rodrigo JA, PonsSanchez C et al. Manual del Sistema de Información Vacunal. Valencia: Conselleria de Sanitat. Generalitat Valenciana; 2009. (Citado el 12/07/15). Disponible en: http://www.sp.san.gva.es/biblioteca/publicacion_dgsp. jsp?cod_pub_ran $=19281 \&$ tacc $=15$

13. Pastor Villalba E, Cremades Bernabeu A, Alguacil Ramos AM, Martin Ivorra R, Portero Alonso A, Lluch Rodrigo JA et al. Recaptación activa de la $1^{\mathrm{a}}$ y $2^{\mathrm{a}}$ dosis de triple vírica en la comunidad valenciana. Utilización y rendimiento de la herramienta del Sistema de Información Vacunal (SIV). $6^{\circ}$ Congreso de la Asociación Española de Vacunología 23 al 26 de Nov 2011. Santiago de Compostela: Asociación Española de Vacunología; 2011.

14. Alguacil-Ramos AM. La farmacovigilancia de vacunas en la Comunidad Valenciana y su impacto en los programas de vacunación. Años 2005-2011. [Tesis doctoral] Valencia: Universidad de Valencia; 2014.

15. Pastor-Villalba E, Martin-Ivorra R, Alguacil-Ramos AM, Portero-Alonso A, Lluch-Rodrigo JA. Exhaustividad de la declaración en el Sistema de Información Vacunal (SIV) de vacunas no incluidas en el calendario sistemático infantil de la comunidad valenciana. Años 2009 a 2012. $7^{\circ}$ Congreso Asociación Española de Vacunología. 25-27 nov 2013. Cáceres: Asociación Española de Vacunología; 2013. 